\title{
LDL uptake and neutrophil effector function
}

\section{Ondracek AS ${ }^{1}$, Hofbauer TM ${ }^{1}$, Kascha L ${ }^{1}$, Feist CWM ${ }^{1}$, Wadowski PP ${ }^{2}$, Mangold A ${ }^{1}$, Lang IM ${ }^{1}$}

1 Department of Cardiology, Internal Medicine II, Medical University of Vienna, Austria

2 Department of Angiology, Internal Medicine II, Medical University of Vienna, Austria

\section{BACKGROUND}

The number of activated neutrophils in the circulation of hyperlipidemic patients is correlated with levels of low density lipoprotein (LDL). ${ }^{1}$ Activated neutrophils can expel their chromatin, forming net-like structures. $^{2}$ These neutrophil extracellular traps (NETs) constitute important risk factors for acute myocardial infarction (AMI). ${ }^{3}$ Pro-protein convertase subtilisin/kexin 9 (PCSK9) is a regulator of LDL receptor (LDLR) expression and has gained attention in the treatment of hyperlipidemia. ${ }^{4}$
AIM

We aimed to investigate the influence of LDL and PCSK9 on neutrophils and NET formation in patients with AMI.

\section{METHODS}

25 consecutive AMl patients were enrolled to assess cardiovascular (CV) risk factors, ex vivo NET formation in response to PCSK9 and ionomycin (IM), and neutrophil surface receptor expression by flow cytometry

\section{RESULTS}

\begin{tabular}{|c|c|c|}
\hline Risk factors & Patients $(\mathbf{n})$ & $\%$ \\
\hline Male sex & 22 & 88.0 \\
\hline Smoker & 13 & 52.0 \\
\hline Diabetes mellitus II & 4 & 16.0 \\
\hline Arterial hypertension & 19 & 79.2 \\
\hline Dyslipidemia & 17 & 70.0 \\
\hline BMI > 25 & 14 & 56.0 \\
\hline
\end{tabular}

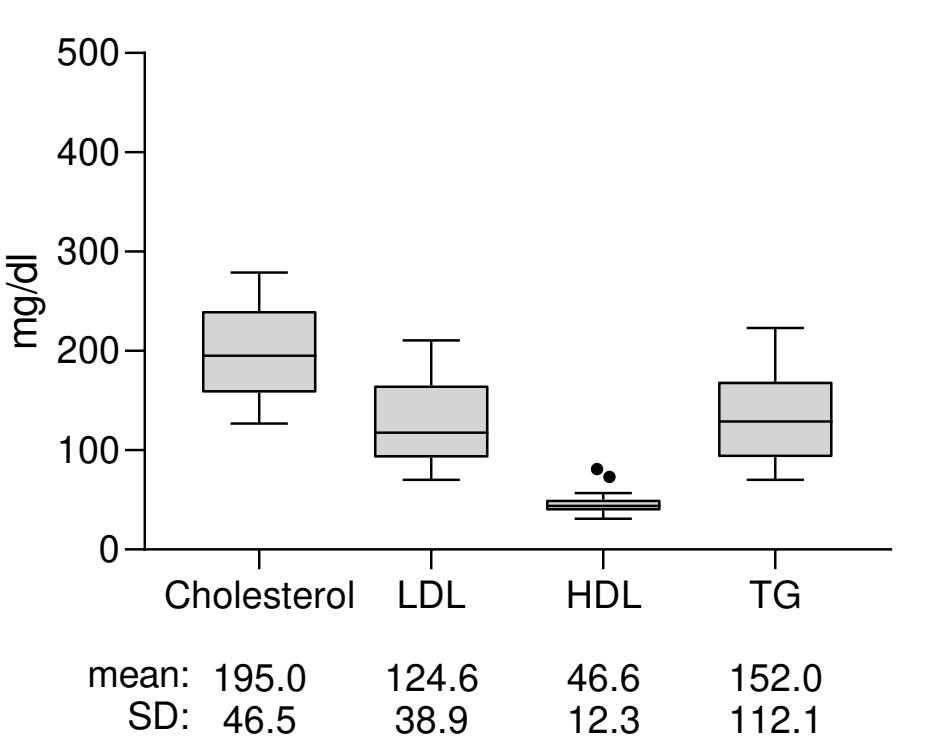

Figure 1. CV risk factors and lipid profile of AMI patients.

a

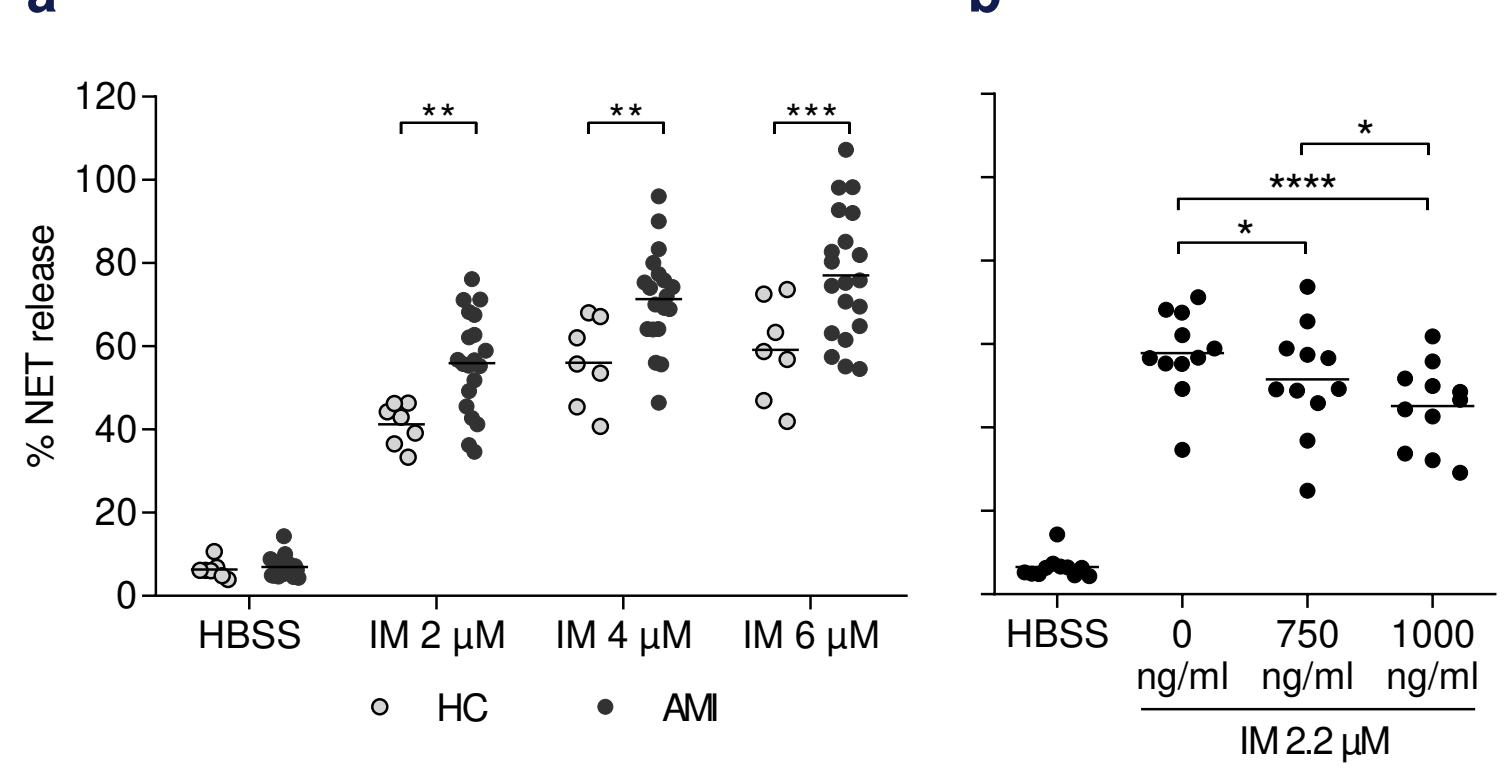

Figure 2. Ionomycin (IM) induced NET formation is (a) higher in AMI patients compared to healthy controls (HC). (b) Pre-conditioning of neutrophils of $\mathrm{AMI}$ patients with PCSK9 attenuates stimulated NET formation in a dose-dependent manner.

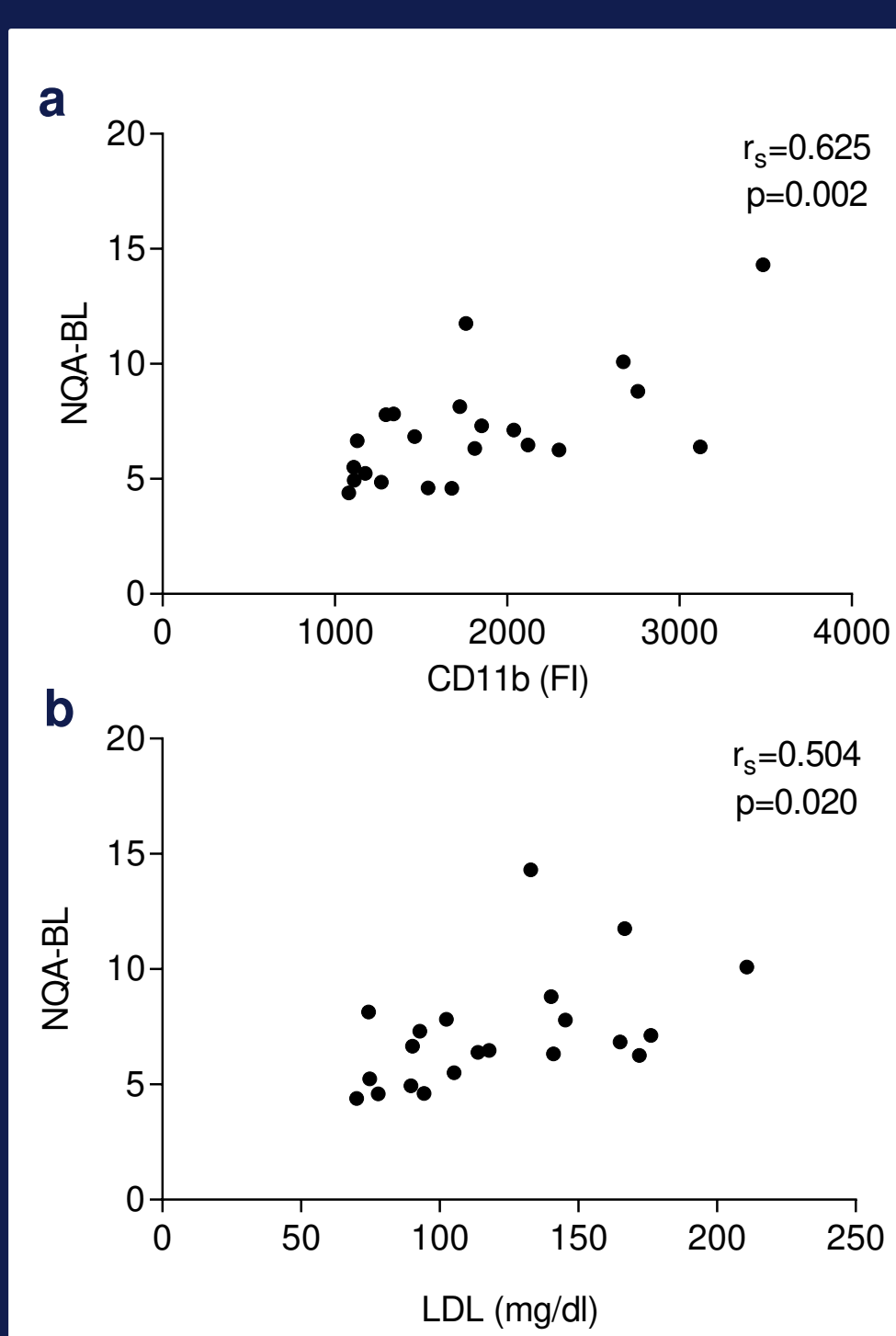

Figure 3. Basal NET formation is correlated with (a) CD11b expression of neutrophils and (b) LDL levels of AMI patients. BL, baseline; NQA, NET quantification

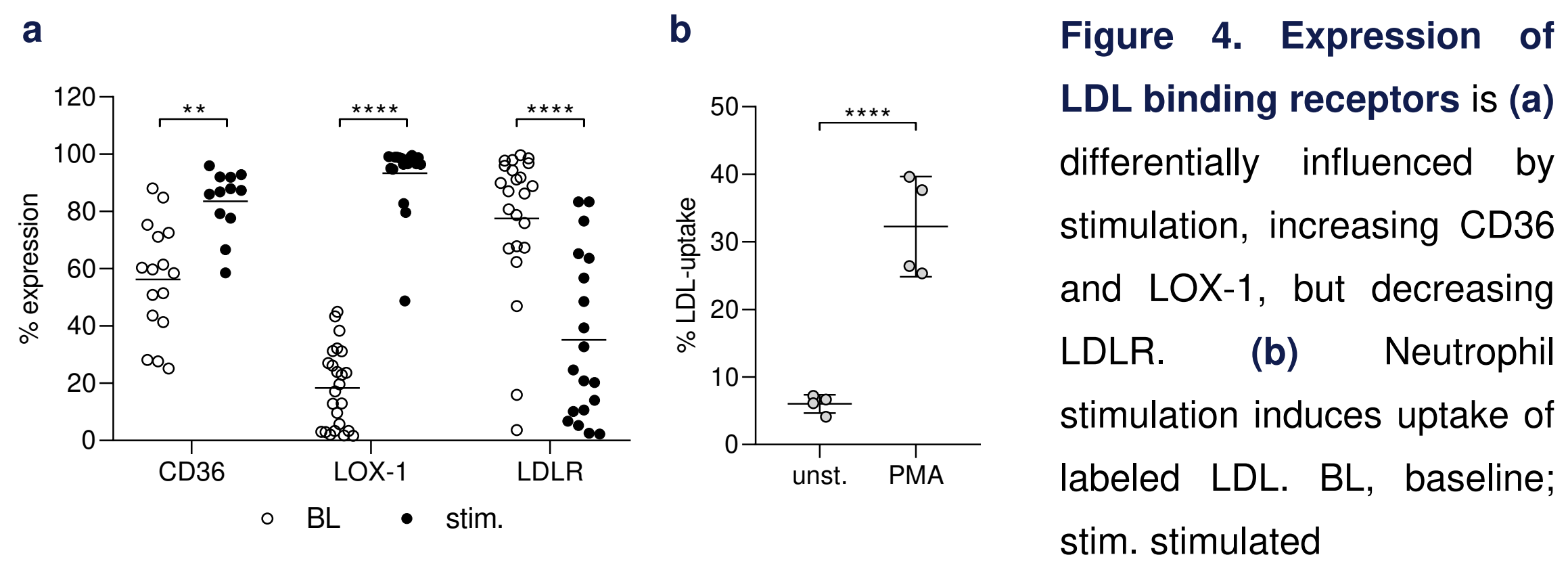

\section{CONCLUSION}

- LDL primes neutrophils, elevating their propensity to undergo NET formation, while PCSK9 reduces NET formation

- Our data indicate a role of LDL uptake for neutrophil effector function during cell activation. 